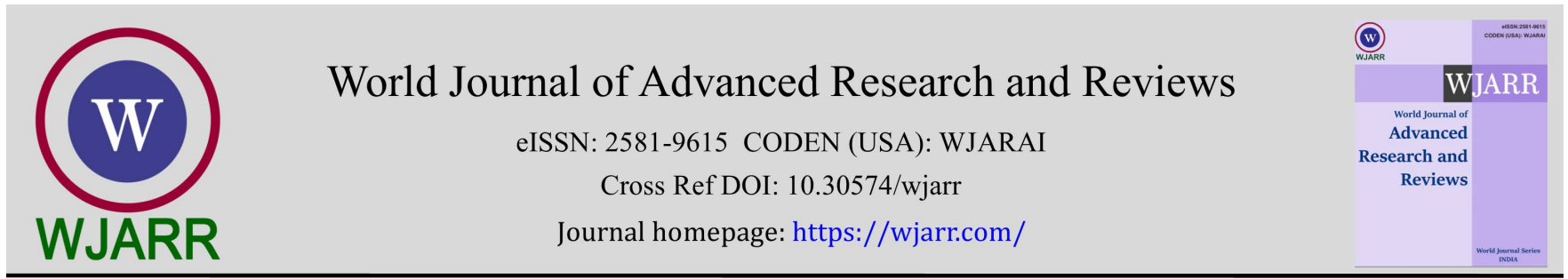

(RESEARCH ARTiClE)

\title{
Chinese music therapy to treat depression in children and adolescents: The BARIT- Model
}

\author{
Wolfgang Mastnak* \\ Beijing Normal University BNU.
}

World Journal of Advanced Research and Reviews, 2022, 13(01), 322-331

Publication history: Received on 08 December 2021; revised on 10 January 2022; accepted on 12 January 2022

Article DOI: https://doi.org/10.30574/wjarr.2022.13.1.0035

\begin{abstract}
Depression is broadly considered a global epidemic. In China it ranks among the most prevalent mental disorders and is seriously affecting the younger generation (prevalence between $4 \%$ and $41 \%$ ), hence the necessity to foster health education and sustainable resilience. Meta-synthetic construction resulted in a music-based model of educational therapy comprising five principles, beauty, activation, responsiveness, immersion and transformation, hence the name 'BARIT-model': (i) referring to anthropological, psychological and neuroscientific aesthetics, the approach benefits from the healing power of aesthetic experience, (ii) behavioural activation is widely regarded as an efficient approach to treat depression. Different from conventional cognitive behavioural therapy, the BARIT-model involves artistic activities such as music improvisation, sound scene improvisation, vocal experiments or creative variations of Chinese martial arts, (iii) responsiveness concerns the qualitative similarity of emotion and music, alongside the patients' feeling of being 'understood' by what they are listening to, (iv) while depressive mood tends to occupy the whole person, music immersion can help to escape that 'pathological cage', (v) finally, traumatic roots of depression need therapeutic processing, such as artistic symbolisation, intermodal transformation and relabelling of traumata as potential source of creative performance. The BARIT-model is part of a comprehensive project to improve mental health in Chinese children and adolescents through arts-based methods for classroom education, which encompasses - in addition to depression - attention deficit hyperactivity disorders, oppositional defiant disorders, anxiety disorders, stress-related disorders and burnout syndromes, eating disorders such as anorexia nervosa or binge eating, as well as disorders related to the COVID-19 pandemic, e.g. developmental syndromes caused by lockdown and social distancing.
\end{abstract}

Keywords: Aesthetic immersion; Chinese music therapy; Clinical music education; Self-regulation techniques; Sound scene improvisation; Translational education

\section{Introduction}

Depression is commonly considered a global burden. Regarding this issue today meets two essential problems: (i) extreme variations of quantitative data raise the question about their reliability and how to estimate true distributions of depressive disorders, (ii) how to assess the influence of the COVID-19 pandemic on mood disorders and how to distinguish between various pathogenic factors of depression, such as genetic dispositions or social distancing. A Chinese study [1] from Changsha and Wuhan discusses these issues:

Depression is a common mood disorder characterized by persistently low mood state, affecting $1.4-37.5 \%$ of the population [...] Several studies had shown that depression not only reduces the quality of life of patients but also increases the risk of chronic physical diseases and suicide [...] Recent studies have shown that the prevalence of depressive symptoms in the general population is high during the COVID-19 epidemic, ranging from 3.7 to $68 \%$ [...] A published meta-analysis study included 14 studies and suggested that $33.7 \%$ of the general population had

\footnotetext{
* Corresponding author: Wolfgang Mastnak

Beijing Normal University BNU.

Copyright (C) 2022 Author(s) retain the copyright of this article. This article is published under the terms of the Creative Commons Attribution Liscense 4.0.
} 
depressive symptoms during the COVID-19 pandemic [...] Additionally, several Chinese studies reported the prevalence of depressive symptoms in the general population during the COVID-19 outbreak. For example, a survey study [...] with 56,679 Chinese participants reported $27.9 \%$ of the general population had depressive symptoms. A previous study from Shenzhen suggested that the prevalence of depressive symptom was $6.2 \%$ in the general population quarantined [...] Another study [...] found the prevalence of depression was approximately $14.6 \%$, with mixed samples including people affected and people unaffected. Therefore, the prevalence of depressive symptoms among the general population is still inconsistent, probably due to the different population and sampling issues in these studies, which deserves further study.

While conventional evidence based medicine mainly focuses on so called levels of evidence and effect sizes, systemic approaches in medicine regard health-related issues in the context of dynamic systems, alongside a clear focus on different factors such as sociocultural or epigenetic parameters. This leads to distinct identification of (i) risk groups and risk conditions such as perinatal depression [2,3] and the suggestions that 'urgent attention is needed to address this public health priority in China' [4], (ii) sociocultural challenges such as the combination of loneliness and depression among rural empty-nest elderly adults [5] or depression among left-behind children in China [6, 7], including perspectives of developmental psychopathology and cultural-clinical psychology of depression [8], (iii) psychosomatic circuitries and interdependencies such as the problem that depression is associated with an increased risk of all-cause and cardiovascular disease (CVD) mortality in adults in China, particularly in men: 'These findings highlight the importance and urgency of depression management as a measure for preventing premature deaths in China' [9] and (iv) the huge systemic and public health costs caused by depression [10].

The present article is dealing with music- and arts-therapeutic means to supports children and adolescents with depression or depressive symptoms in China, hence the focus on the prevalence of depression in the Chinese younger generation. A meta-analysis [11] on this issue shows that depressive symptoms are common in children and adolescents, but qualifies that the prevalence of depressive symptoms in children and adolescents in China vary significantly across studies, hence the necessity for a critical meta-analysis of relevant studies in English (PubMed, PsycINFO and EMBASE) and Chinese (China National Knowledge Internet, WANFANG Data and SinoMed) databases:

Random-effects model was used to synthesize the prevalence of depressive symptoms. Eighteen studies covering 29,626 participants were identified and analyzed. All these studies used the same measurement to identify depressive symptoms. The reported point prevalence of depressive symptoms ranged between $4 \%$ and $41 \%$ in the studies; the pooled prevalence of depressive symptoms was 19.85\% (95\% confidence interval: 14.75\%-24.96\%). In the subgroup analyses the prevalence of depressive symptoms was significantly associated with the region where the study was conducted: $17.8 \%$ in eastern, $23.7 \%$ in central, $22.7 \%$ in western, and $14.5 \%$ in northeast regions of China $(\mathrm{P}<0.001)$. Considering the adverse impact of depressive symptoms on health outcomes, regular screening and effective interventions should be implemented in this population.

Another meta-analysis [12] assessed the trend of depressive symptoms in China among adolescents from 1988 to 2018 , called depressive symptoms a pervasive mental health problem in Chinese adolescents, and showed that the prevalence of depressive symptoms has generally increased over time [13]: The prevalence estimates before 2000 were $18.4 \%$ and $26.3 \%$ after 2016. The pooled prevalence of depressive symptoms among children and adolescents was estimated 22.2\%. Moreover, research suggests that this increasing trend interdepends with social environments.

This alarming amount of depression and depressive syndromes in Chinese children and adolescents makes the disease also an educational issue. According to a meta-analysis [14] including 42,374 Chinese primary school pupils, the pooled prevalence of depressive symptoms is $17.2 \%$. Moreover the authors pointed out that prevalence levels significantly varied between geographic regions, with western China reporting the highest rates.

A meta-analysis [15] assessing the prevalence of depressive symptoms among adolescents in secondary schools in mainland China revealed a pooled prevalence of depressive symptoms of $24.3 \%$ and showed, similar to the study on depression in primary education, high between-studies heterogeneity which ranged from $6.2 \%$ to $64.8 \%$. The authors reported that there was an increased prevalence with increasing grades from year 1 in junior secondary school (24.5\%) to year 3 in senior secondary school (40.1\%).

Today the mental health status of children and adolescents in China is importantly influenced by the COVID-19 pandemic, which particularly increases depression, anxiety disorders and posttraumatic syndromes. A large sample study [16] on the psychological impact of the COVID-19 epidemic on adolescents in China estimated the prevalence of depression (35.9\%), anxiety (28,9\%), and posttraumatic growth PTG (45.6\%) and showed through regression analysis that female sex and older age were associated with higher levels of depression and anxiety, and lower levels of PTG: 
Symptoms related to COVID-19, excessive attention to epidemic information, living in urban or severe epidemic areas, and conflicts with parents during home quarantine were risk factors for depression, anxiety, and PTG. Frequent communication with peers, exercise, and receiving public welfare psychological assistance were protective factors. Poor family economic status was a significant risk factor for depression and PTG [...] The factors associated with psychological problems and PTG provide strategic guidance for maintaining adolescents' mental health in China and worldwide during any pandemic such as COVID-19.

The present study's intentions coincide with these aims: to enhance support for the mental health of children and adolescents during the COVID-19 era and the post-COVID-19 era, as well as any following pandemic, and to provide culturally sensitive models of arts-based education and therapy both in China and internationally.

\section{Aims and Methods}

The main purpose of this study was to develop and provide systemic music therapeutic methods for Chinese classroom education to alleviate depression and depressive syndromes in children and adolescents. Although previous clinical and special educational experiences shed light on the efficacy of specific musical interventions, there are two deficiencies that inspired the present investigations.

Firstly, certain influential circles in the realm of music therapy are mainly based on general models such as NordoffRobbins music therapy, which goes back to the late 1950s [17], or Guided Imagery and Music, a method to evoke emotions and access the unconscious [18]. These models are well-defined, but not tailored to clear diagnoses such as depression, hence the need for more distinct music therapeutic approaches.

Secondly, standardised methods of evidence based medicine such as randomised controlled trials (RCT) or metaanalyses of RCTs are broadly accepted means to estimate effect sizes through inferential statistics. Nonetheless, they inherently ignore correspondence-theoretical distances between the actual object and the corresponding mathematical model, a shortcoming that may considerably affect their reliability, hence the two novel parameters of coherence size and confidence range [19] - notions that also influenced the present study.

Moreover, rigid structures of research designs such as RCTs require standardised non-complex interventions and simple data-dimensions. Consequently, corresponding studies frequently associate intervention and control groups with 'liked music' and 'disliked music' or categorisations such as 'happy music', 'sad music' and 'angry music', which is - from a musicological and aesthetic perspective - far too simplistic. Although these studies may fulfil standardised requirements of evidence based medicine, their inner inadequacy goes hand in hand with epistemological weaknesses, hence the likelihood of irrelevant or false results.

Neither RCTs nor meta-analyses or systematic reviews are able to inspire and generate new therapeutic interventions. Regarding that the core of the present study was to develop new music therapeutic models for the treatment of depression and depressive syndromes, other scientific approaches were needed, hence the choice of comparative research and deductive reasoning, alongside the principles of systemic meta-syntheses [20].

Key principles of systemic meta-syntheses go back to in-depth analyses of history of science and the evolution of seminal theories in psychology, psychosomatics and medicine, which witness the power of epistemological syntheses of all sorts of ideas and discoveries and shed light on ground-breaking concepts and innovation. Broadly speaking: great minds discovered the inner logic of data from clinical experience, subjective theories and interdisciplinary sciences and created consistent frameworks - and this is precisely the way, systemic meta-syntheses are built.

This also explains the use of the word 'systemic'. Given that tangible entities have their very unique characteristics, holistic truth-philosophical representation strives for a most adequate isomorphism between the actual object in the real world and its theoretical representation in the realm of science. Distinct from research on single perspectives, dimensions or parameters, systemic meta-syntheses try to take the complexity of phenomena into account, hence the term 'systemic'.

In general, there are two different ways to create a systemic meta-synthesis: a bottom-up and a top-down mode. In bottom-up mode studies on a given issue or thematic field are taken into account and explorative comparative processing tries to trace down general principles as well as their inner logic and coherence. This process generates hypotheses, which usually appear more robust than results of single hypothesis generating studies. 
The top-down mode starts with a given hypothesis and goes on searching for confirmatory or contradictory evidence. While the whole process reminds of conventional hypothesis testing, the 'timeline inversion' makes the decisive difference: What supports or destroys the hypothesis is derived from previous studies. The advantage of this approach lies in the enormous pool of relevant data, which have to be re-evaluated though.

In addition to these two main types, the notion 'systemic meta-synthesis' encompasses all approaches (i) which respect epistemology and philosophy of sciences and (ii) integrate data and results from various types of studies (iii) to create a novel theory or theoretical framework. And there are basically no discipline-related limitations or apodictic exclusion criteria.

\section{Results and Discussion}

Referring to a broad pool of relevant studies, meta-synthetic construction as described above resulted in a music-based framework for health education and treatment of depression and depressive syndromes in classroom-education. The BARIT-model shall be understood as an open dynamic system that suggests adaptation to specific educational conditions and encourages further theoretical studies as well as ongoing development of appropriate techniques, alongside interdisciplinary exchange.

\subsection{Beauty}

The sense of beauty is a unique feature of the human mind and also the title of a famous book on aesthetics [21], where the author, philosopher George Santayana (1863 - 1952), defined beauty as an 'objectified pleasure'. Quite close to modern perspectives in psychological aesthetics, he argued that beauty arises when the emotion of pleasure intertwines with specific qualities of an object, and he highlighted that 'the sense of beauty has a more important place in life than aesthetic theory has ever taken in philosophy'.

Today, aesthetics define an interdisciplinary domain that encompasses artistic views, philosophical positions, psychological approaches and neuroscientific research. In this context - and with significant relevance to the BARITmodel - cross-cultural empirical aesthetics [22] distinguish between cultural impacts on the experience of beauty and universal psychological processes underlying aesthetic preference, arguing that aesthetic preference emerges from basic perceptual and valuation processes 'that are common to all humans, and to many other animals'.

From a genuine neuroscientific perspective, neuroaesthetics analyse likewise the art's diversity and universality [23] and the triptych of 'art, science and brain' [24] goes hand in hand with interdisciplinary approaches. Using neurophysiological techniques, neuroimaging and network science algorithms [25], they give insights into the functional connectivity between brain regions underlying our aesthetic experiences, alongside relevant neural correlates [26]. These studies importantly substantiate music-therapeutic models to treat depression and allow the construction of interdisciplinary frameworks.

Discussing beauty as a means to treat depression raises the crucial question whether depressive conditions actually allow the experience of beauty - and while since the early times of aesthetic research intense pleasure proved its tendency to generate experience of beauty, a more recent study [27] focused on the inverse: Is intense pleasure necessary for intense beauty? Regarding self-reports of anhedonia, mood states and depression, the authors concluded that both general and anhedonic results supported the claim that intense beauty requires intense pleasure. However, this study used images and not music, which might act as a more intensive stimulus. By way of illustration, Teresa Boning [28] reported the case of a highly suicidal female patient diagnosed with major depression who made significant progress in her vocal quality. After a clinical singing lesson she referred to her performance and stated: 'It is impossible to kill such a beauty' - and this was the decisive watershed in her therapy.

In clinical fields we are confronted with another, seemingly paradoxical phenomenon: the beauty of depression. However, the more we explore this area, the more we get aware of its enormous range and multifaceted features, which is also substantiated by neuroscientific findings about joyful beauty and sorrowful beauty [29]. We find notions such as 'the beauty of being depressed', the contemporary Zen-Koan 'depression is beautiful' and - not surprisingly - the beauty of sad music [30], which is closely interrelated with what is called 'responsiveness' below: the intense experience of a qualitative analogy between one's depressive feelings and music. Such 'cases' require both mindful and interdisciplinary approaches, which may qualify mono-dimensional therapeutic goals.

Although complementary medical approaches highlight that visual arts, poetry, dance or music can have 'a major impact on patients' spiritual well-being and health' [31], medicine is usually reluctant to call beauty a therapeutic agent. 
Notwithstanding these reservations, we argue that clinical observations suggest a certain healing potential of beauty, particularly in depression, hence the call for relevant interdisciplinary research. Related considerations also involve philosophical aesthetics and the ontology of beauty - and a critical assessment of the modern arts highlighted 'Die heilende Kraft der Schönheit' [The healing power of beauty] [32]. In (music) educational areas, beauty does not relate to operationalised techniques, but is rather interconnected with principles of artistic discovery and self-experience, healing purposes included.

\subsection{Activation}

Behavioural activation is widely regarded as one of the most efficient interventions to treat depression - and it also an important component of cognitive-behavioural therapy (CBT), which ranks among the standard treatments of depression. Today, technology is increasingly used in the context of CBT for depression, and multimedia as well as hybrid CBT might be as effective as face-to-face CBT [33].

Calling cognitive behavioural therapy the best-evidenced therapy for depression, a study from the medical school of the University of Exeter in the UK [34] referred to its high cost and suggested that 'a simpler therapy - behavioural activation (BA) - might be as effective and cheaper than is CBT' and discussed the clinical efficacy and cost-effectiveness of BA compared with CBT for adults with depression.

Behavioural activation also includes physical exercise, which is an effective intervention for depression [35], also when depression is a comorbid condition such as in individuals with multiple sclerosis [36]: 'Behavioral activation may represent one mechanism by which physical activity improves depression in MS. Future physical activity trials should capitalize on this relationship and place additional emphasis on identifying and engaging in personally meaningful life activity'. This last remark 'meaningful life activity' is of crucial importance for our approach and also involves perspectives of lifestyle medicine in depression [37].

Activation is a cornerstone of the BARIT-model and involves both principles and practice. The principles make this approach different from merely 'prescribed activity' and includes (i) mindful selection, (ii) subjective significance and (iii) sensed identity - in other words, the activity shall be experienced as a natural and substantial part of one's individual life. Selecting activities might need time, support and self-exploration and can be regarded as an integral part of therapy or health education.

Possible practices may include ensemble improvisation, sound scene improvisation [38] such as in Polyaesthetic Education, individual vocal experiments, sound-light installations or creative variations of traditional Chinese martial arts such as 五离戏 wǔqínxì. This model traces back to the Eastern Han Dynasty, is known as the 'five animals' movements imitate tigers, deer, bears, apes and birds - and also used in other therapeutic areas such as the metabolic syndrome [39], which also may interrelate with depression [40].

\subsection{Responsiveness}

Loneliness is a significant variable affecting depression [41]. From a psychopathological perspective, loneliness is a distinct negative emotion, which has to be clearly distinguished from social isolation or eremitical solitude or eremitical life. A European study on the experience of loneliness among young people with depression [42] focused particularly on maladaptive cognitions about one's role in the social world and concluded that young people with depression experience loneliness as an insurmountable distance between themselves and others. In this context, the authors identified non-disclosure of depression and the debilitating nature of depressive symptoms as factors perpetuating a vicious cycle of loneliness and depression, hence their suggestion that approaches to tackling the problem might include helping young people communicate about their depression 'to trusted friends and educating their social networks in how to support them'. In the context of the present article this suggestion also involves health education and psychoeducation at school.

Loneliness is not necessarily associated with social isolation, but very often caused by the feeling of being misunderstood. In Psychology Today, Jonathan Rottenberg [43] said that people who face depression must be prepared for at least two long journeys: 'One journey is to face depression itself. The symptoms of depression - despondency, lethargy, nightly insomnia, an inability to concentrate - are painful and difficult to manage. The second journey, is in many ways, harder, and often longer, which is to face other people's misunderstandings of their depression, misunderstandings that are sometimes insulting, confusing, and often unhelpful in getting the symptoms under control'.

Clinical music therapeutic experience gives evidence that individuals with depression may feel understood by music and create an 'aesthetic bond' which seems to depend on several crucial conditions: (i) music mirrors the patient's 
emotional quality without using verbal - an thus abstract - codes. By way of illustration, a musical phrase expressing desire is instinctively closer to one's experienced desire than the word 'desire', (ii) music does not judge or give comments and advices, but provides a tacit understanding, and (iii) music creates an emotional sphere which considerably differs from the fragile and transitory shallowness of social dynamics.

However, in the health-educational understanding of BARIT, responsiveness is not limited to this empathetic soundshelter-function, but meant to initiate a curative sequence: (i) one's intimate 'sympathetic music' gives confidence and evokes feelings of safety, as well as being empathically understood, (ii) self-discovery of the characteristics and underlying processes of feeling understood by music, as well as a hidden/suppressed longing for being understood by peers or close friends, (iii) application of what has been learned through the first two phases in social contexts, educational and symbolic areas included, and (iv) development of artistic ways of communication about the whole range of emotions, depressive ones included.

\subsection{Immersion}

Depression may downright obsess a patient - an experience that often goes hand in hand with an egocentric perspective and sharply differs from its counterpart, the observer's view. In cognitive sciences these two poles are called immersion and distancing, and a study on emotion-focused therapy (EFT) of depression [44] pointed out that significant differences across sessions were only found in the good-outcome cases which showed a significant decrease of immersion and an increase of distancing, and this evolution pattern was found related to the reduction of symptoms. Moreover, at the beginning of therapy, distancing was higher in the poor-outcome cases rather than in the good-outcome cases, hence the authors' conclusion that progressive and significant evolution from higher immersion at the initial phase to higher distancing in the final phase may be helpful in EFT for depression.

As depression occupies the patient, it replaces in a sense his personality, which may even result in the (in German speaking countries clinically known) expression 'I am depression' ['Ich bin eine Depression'] instead of 'I have depression'. While depression is associated with decreased self-worth, less was known about how depression relates to the degree of perceived unity of the self over time, called 'continuous identity' - until a study [45] showed that even people with depressed mood have an instinctive grasp of the possibility to an improved future self-worth despite the negative cognitions associated with present self-worth and hopeless expectations about the future. While depressed and hopeless individuals may view the world negatively and feel hopeless about their general future, these results suggest that depressed individuals distinguish between hopelessness about future external success and future selfimprovement. Despite perceiving their past and future selves to be more positive, depression severity was associated with less continuous identity. Since depressed individuals perceive a future self as a return to or a recovery of a past self, therapeutic strategies may focus on improving a sense of continuous identity with past and future selves, as well as on deriving meaning from current life difficulties to improve beyond a past self, in other words, growing to a superior future self.

This aspect basically concerns the BARIT-model and raises the question: How to reach that? The present study suggests a mediating link: music. Dominating experience of being haunted by depression which perceptibly banishes one's selfidentity is likely to cause a loss of self-awareness, alongside pathological sequelae. Supporting fragmented selves, mindful ways in holistic healthcare are about to gain importance, spiritual immersion [46] included. The BARIT-model encompasses musical immersion, either through listening or music-making. Both modes differ sharply from casual perception of background music or mechanical exercises, and require inner readiness to get absorbed by the aesthetic experience of sound and rhythm. Intense music immersion can replace the dominating depressive mood and facilitate one's way back to the 'true self' - a notion, which also requires philosophical reflection.

Individuals reported three main experiences of music immersion, which can invert depression or make it even vanish into thin air: (i) a mystical union of music and the self, akin to love fusion or moments when the soul is made one with God, (ii) a moment of aesthetic joy in which the obsessive-compulsive power of depression falls apart, alongside a feeling of liberation and redemption, and (iii) the revival of the feeling of being alive and genuine self-actualisation, in contrast to being permanently beset with sadness, hopelessness and inner emptiness.

\subsection{Transformation}

Human resilience is widely regarded as a complex and highly efficient inborn capacity to deal with difficult situations and enhance appropriate self-adjustment, not least in the sense of interconnected health and social systems [47] - and we observe children's incredible ability to grow within extremely adverse and challenging environments [48]. Anthropology and psychology shed light on this evolutionary phenomenon and try to explain underlying mechanisms. 
Nonetheless, their roots seem to go hand in hand with the nature of the human race and call for further interdisciplinary studies.

Resilience and coping abilities are inextricably intertwined [49] and paediatric psychiatry emphasises the importance of supportive environments in which children can 'develop healthy coping mechanisms' [50] - and research on what coping mechanisms actually are gains importance. We enter an interdisciplinary field which comprises highly different approaches such as psychodynamic analyses of voluntary and involuntary coping mechanisms [51] or neuropsychological findings explaining how the brain regulates stress-responsive structures when coping behaviours are engaged [52].

In the realm of arts-based therapies, clinical expressive arts therapy [53] highlights the therapeutic power of creative expression, which also relates to coping mechanisms and artistic ways of trauma processing. Being one of the great minds in this area, Paolo Knill [54] suggested an intermodal approach. Referring to philosophical perspectives of phenomenology, hermeneutics and deconstructionism, he importantly spoke of 'indigenous healing systems' and designed (implicitly) a novel model of arts-therapeutic system theory. Discussing the essence of arts therapies with the author of this article, he often referred to his concept of the 'incommunicable third' as something that emerges unforeseen from out of a therapeutic encounter and highlighted the therapeutic potential of intermodal creative transformation such as, for instance, expressing a traumatic experience through dance, which consequently inspires a poem that leads to music improvisations or expressive painting, and so on.

Akin to Knill's theory, the BARIT-model understands (intermodal) transformation as a symbol-oriented process involving (i) a non-cognitive discovery of the sources of one's depressive feelings, such as the pathogenic interaction between stimuli - using the term 'trauma' requires the corresponding stimulus's negative interpretation or painful perception - and self-images resulting in fragmentation of one's identity, (ii) a primary transformation of these triggers' into artistic shapes and (iii) further creative 'trauma processing' leading to a characteristic triple function: mindful acceptance of the traumatic experience as a biographical givenness - inversion of its traumatic power through artistic 'domination' - discovery of the formerly detrimental stimulus as a source of personal growth and artistic spirit.

\section{Conclusion}

This model-generating study is part of a comprehensive Chinese project to improve mental health in children and adolescence and to alleviate syndromes through arts-based interventions in educational areas. The relevant spectrum includes, according to Chinese psychiatric epidemiology and mental public health studies, most prevalent conditions in the Chinese younger generation, i.e. in addition to depression also attention deficit hyperactivity disorder, oppositional defiant disorder, anxiety disorders, stress and burnout syndromes, eating disorders, and mental disturbances caused by measures to control the COVID-19 pandemic such as social isolation.

Next steps include qualitative exploratory studies, studies to generate standardised models and quantitative multicentre studies, as well as feasibility studies which shall help to provide these educational-therapeutic facilities throughout the People's Republic of China. Moreover, cross-cultural studies shall help to adapt them in different cultures and thus contribute to intermodal culturally sensitive creative and aesthetic therapies.

\section{Compliance with ethical standards}

\section{Funding}

No funding to declare.

\section{Disclosure of conflict of interest}

The author declares no conflict of interest.

\section{Statement of ethical approval}

No approval needed for this type of research. 


\section{References}

[1] Peng S, Lai X, Du Y, Li Y, Tian K, Gan Y. Prevalence and associated factors for depressive symptomatology in Chinese adults during COVID-19 epidemic. Frontiers in Psychology. 2020; 11: 616723.

[2] Yin J, Nisar A, Waqas A, Guo Y, Qi WL, Wang D, Rahman A, Li X. Psychosocial interventions on perinatal depression in China: A systematic review and meta-analysis. Journal of Affective Disorders. 2020; 271: 310-327.

[3] Shi P, Ren H, Li H, Dai Q. Maternal depression and suicide at immediate prenatal and early postpartum periods and psychosocial risk factors. Psychiatry Research. 2018; 261: 298-306.

[4] Nisar A, Yin J, Waqas A, Bai X, Wang D, Rahman A, Li X. Prevalence of perinatal depression and its determinants in Mainland China: A systematic review and meta-analysis. Journal of Affective Disorders. 2020; 277: 1022-1037.

[5] Wang G, Hu M, Xiao SY, Zhou L. Loneliness and depression among rural empty-nest elderly adults in Liuyang, China: a cross-sectional study. BMJ Open. 2017; 7(10): e016091.

[6] Cheng J, Sun YH. Depression and anxiety among left-behind children in China: a systematic review. Child: Care, Health and Development. 2015; 41(4): 515-523.

[7] He B, Fan J, Liu N, Li H, Wang Y, Williams J, Wong K. Depression risk of 'left-behind children' in rural China. Psychiatry Research. 2012; 200(2-3): 306-12.

[8] Ryder AG, Sun J, Zhu X, Yao S, Chentsova-Dutton YE. Depression in China: integrating developmental psychopathology and cultural-clinical psychology. Journal of Clinical Child and Adolescent Psychology. 2012; 41(5): 682-694.

[9] Meng R, Yu C, Liu N, He M, Lv J, Guo Y, Bian Z, Yang L, Chen Y, Zhang X, Chen Z, Wu T, Pan A, Li L; China Kadoorie Biobank Collaborative Group. Association of depression with all-cause and cardiovascular disease mortality among adults in China. JAMA Network Open. 2020; 3(2): e1921043.

[10] Hsieh CR, Qin X. Depression hurts, depression costs: The medical spending attributable to depression and depressive symptoms in China. Health Economics. 2018; 27(3): 525-544.

[11] Rao WW, Xu DD, Cao XL, Wen SY, Che WI, Ng CH, Ungvari GS, He F, Xiang YT. Prevalence of depressive symptoms in children and adolescents in China: A meta-analysis of observational studies. Psychiatry Research. 2019; 272: 790-796.

[12] Li JY, Li J, Liang JH, Qian S, Jia RX, Wang YQ, Xu Y. Depressive Symptoms Among Children and Adolescents in China: A Systematic Review and Meta-Analysis. Medical Science Monitor. 2019; 25: 7459-7470.

[13] Su Q, Liu G. Depression in Chinese adolescents from 1989 to 2018: An increasing trend and its relationship with social environments. Current Psychology. 2020.

[14] Xu DD, Rao WW, Cao XL, Wen SY, An FR, Che WI, Bressington DT, Cheung T, Ungvari GS, Xiang YT. Prevalence of depressive symptoms in primary school students in China: A systematic review and meta-analysis. Journal of Affective Disorders. 2020; 268: 20-27.

[15] Tang X, Tang S, Ren Z, Wong DFK. Prevalence of depressive symptoms among adolescents in secondary school in mainland China: A systematic review and meta-analysis. Journal of Affective Disorders. 2019; 245: $498-507$.

[16] Zhou J, Wang Y, Bu T, Zhang S, Chu H, Li J, He J, Zhang Y, Liu X, Qiao Z, Yang X, Yang Y. Psychological impact of COVID-19 epidemic on adolescents: a large sample study in China. Frontiers in Psychiatry. 2021; 12: 769697.

[17] Kim Y. The early beginnings of Nordoff-Robbins music therapy. Journal of Music Therapy. 2004; 41(4): $321-339$.

[18] Beebe LH, Wyatt TH. Guided imagery and music: using the Bonny method to evoke emotion and access the unconscious. Journal of Psychosocial Nursing and Mental Health Services. 2009; 47(1): 29-33.

[19] Mastnak W. Coherence size and confidence range: two new parameters in psycho-cardiology. Frontiers of Cardiology \& Cardiovascular Medicine. 2021; 1(1): 1-17.

[20] Mastnak W. Systemic Meta-Synthesis. ResearchGate. 2021.

[21] Santayana G. The Sense of Beauty: Being the Outlines of Aesthetic Theory. New York: C. Scribner's sons. 1896.

[22] Che J, Sun X, Gallardo V, Nadal M. Cross-cultural empirical aesthetics. Progress in Brain Research. 2018; 237: 77103. 
[23] Nadal M, Chatterjee A. Neuroaesthetics and art's diversity and universality. Wiley Interdisciplinary Reviews. Cognitive Science. 2019; 10(3): e1487.

[24] Zeki S, Bao Y, Pöppel E. Neuroaesthetics: The art, science, and brain triptych. PsyCh Journal. 2020; 9(4): 427-428.

[25] Reybrouck M, Vuust P, Brattico E. Brain connectivity networks and the aesthetic experience of music. Brain Sciences. 2018; 8(6): 107.

[26] Reybrouck M, Vuust P, Brattico E. Neural correlates of music listening: Does the music matter? Brain Science. 2021; 11(12): 1553.

[27] Brielmann AA, Pelli DG. Intense beauty requires intense pleasure. Frontiers in Psychology. 2019; 10: 2420.

[28] Boning T. Funktionale Gesangspädagogik im psychiatrischen Setting [Functional vocal education in psychiatric settings]. PhD-dissertation. Salzburg: University Mozarteum. 2019.

[29] Ishizu T, Zeki S. The experience of beauty derived from sorrow. Human Brain Mapping. 2017; 38(8): 4185-4200.

[30] Pelowski M, Ishizu T, Leder H. Sadness and beauty in art - Do they really coincide in the brain? Comment on "An integrative review of the enjoyment of sadness associated with music" by Eerola et al. Physics of Life Reviews. 2018; 25: 124-127.

[31] Ettun R, Schultz M, Bar-Sela G. Transforming pain into beauty: on art, healing, and care for the spirit. Evidencebased Complementary and Alternative Medicine. 2014; 789852.

[32] Böhm AK. Die heilende Kraft der Schönheit. Eine Kulturkritik der modernen Künste. Amerang: Crotona Verlag. 2000.

[33] López-López JA, Davies SR, Caldwell DM, Churchill R, Peters TJ, Tallon D, Dawson S, Wu Q, Li J, Taylor A, Lewis G, Kessler DS, Wiles N, Welton NJ. The process and delivery of CBT for depression in adults: a systematic review and network meta-analysis. Psychological Medicine. 2019; 49(12): 1937-1947.

[34] Richards DA, Ekers D, McMillan D, Taylor RS, Byford S, Warren FC, Barrett B, Farrand PA, Gilbody S, Kuyken W, O'Mahen H, Watkins ER, Wright KA, Hollon SD, Reed N, Rhodes S, Fletcher E, Finning K. Cost and outcome of behavioural activation versus cognitive behavioural therapy for depression (COBRA): a randomised, controlled, non-inferiority trial. Lancet. 2016; 388(10047): 871-880.

[35] Kvam S, Kleppe CL, Nordhus IH, Hovland A. Exercise as a treatment for depression: A meta-analysis. Journal of Affective Disorders. 2016; 202: 67-86.

[36] Turner AP, Hartoonian N, Hughes AJ, Arewasikporn A, Alschuler KN, Sloan AP, Ehde DM, Haselkorn JK. Physical activity and depression in MS: The mediating role of behavioral activation. Disability and Health Journal. 2019; 12(4): 635-640.

[37] Sarris J, O'Neil A, Coulson CE, Schweitzer I, Berk M. Lifestyle medicine for depression.BMC Psychiatry. 2014; 14: 107.

[38] Roscher W, Thomas C. Sound Scene improvisation as a theatre of signs. In: Roscher W, Gesellschaft für Polyästhetische Erziehung eds. Multiperceptual Consciousness - A selection of five years 'contributions. Wien: Österreichischer Kunst- und Kulturverlag. 1991.

[39] Zou L, Zhang Y, Sasaki JE, Yeung AS, Yang L, Loprinzi PD, Sun J, Liu S, Yu JJ, Sun S, Mai Y. Wuqinxi Qigong as an alternative exercise for Improving risk factors associated with metabolic syndrome: a meta-analysis of randomized controlled trials. International Journal of Environmental Research and Public Health. 2019; 16(8): 1396.

[40] Marazziti D, Rutigliano G, Baroni S, Landi P, Dell'Osso L. Metabolic syndrome and major depression. CNS Spectrums. 2014; 19(4): 293-304.

[41] Erzen E, Çikrikci Ö. The effect of loneliness on depression: A meta-analysis. International Journal of Social Psychiatry. 2018; 64(5): 427-435.

[42] Achterbergh L, Pitman A, Birken M, Pearce E, Sno H, Johnson S. The experience of loneliness among young people with depression: a qualitative meta-synthesis of the literature. BMC Psychiatry. 2020; 20(1): 415.

[43] Rottenberg J. Misunderstood depression: the human toll. Stories of misunderstood depression. Psychology Today. 2010. 
[44] Barbosa E, Silva S, Pinto-Gouveia J, Salgado J. How and when immersion and distancing are useful in emotion focused therapy for depression. Psychotherapy Research. 2019; 29(6): 737-751.

[45] Sokol Y, Serper M. Temporal self-appraisal and continuous identity: Associations with depression and hopelessness. Journal of Affective Disorders. 2017; 208: 503-511.

[46] Glicksohn J, Ben-Soussan TD. Immersion, absorption, and spiritual experience: some preliminary findings. Frontiers in Psychology. 2020; 11: 2118.

[47] Almedom AM. Understanding human resilience in the context of interconnected health and social systems: Whose understanding matters most? Ecology and Society. 2015; 20(4): 40.

[48] Bonanno GA, Mancini AD. The human capacity to thrive in the face of potential trauma. Pediatrics. 2008; 121(2): 369-375.

[49] Friedberg A, Malefakis D. Resilience, trauma, and coping. Psychodynamic Psychiatry. 2018; 46(1): 81-113.

[50] Iacona J, Johnson S. Neurobiology of trauma and mindfulness for children. Journal of Trauma Nursing. 2018; 25(3): 187-191.

[51] Vaillant GE. Involuntary coping mechanisms: a psychodynamic perspective. Dialogues in Clinical Neuroscience. 2011; 13(3): 366-370.

[52] Baratta MV, Maier SF. New tools for understanding coping and resilience. Neuroscience Letters. 2019; 693: 5457.

[53] Goren-Bar A. Clinical Expressive Arts Therapy in Theory and Practice: Psychodynamic Snapshots. Newcastle: Cambridge Scholars Publishing. 2019.

[54] Knill P. Das unvermittelbare Heilmittel oder das Dritte in der Kunsttherapie. In: Peterson P. ed. Ansätze kunsttherapeutischer Forschung. Berlin: Springer Verlag. 1990. 